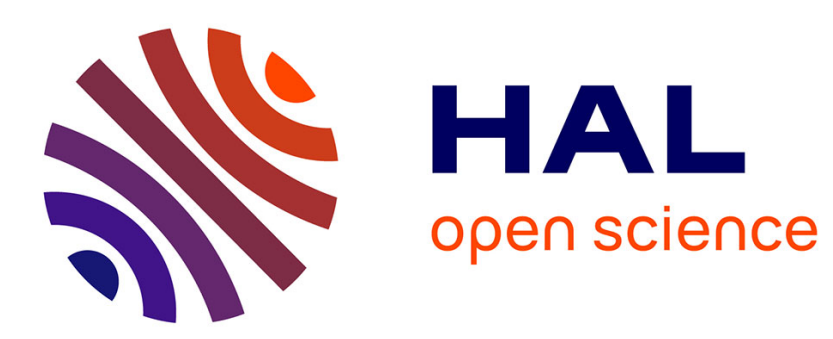

\title{
Electromagnetic fluctuations in the vicinity of the magnetopause
}

\author{
L. Rezeau, S. Perraut, A. Roux
}

\section{To cite this version:}

L. Rezeau, S. Perraut, A. Roux. Electromagnetic fluctuations in the vicinity of the magnetopause. Geophysical Research Letters, 1986, 13, pp.1093-1096. 10.1029/GL013I011P01093 . hal-00408547

\section{HAL Id: hal-00408547 \\ https://hal.science/hal-00408547}

Submitted on 18 Mar 2021

HAL is a multi-disciplinary open access archive for the deposit and dissemination of scientific research documents, whether they are published or not. The documents may come from teaching and research institutions in France or abroad, or from public or private research centers.
L'archive ouverte pluridisciplinaire HAL, est destinée au dépôt et à la diffusion de documents scientifiques de niveau recherche, publiés ou non, émanant des établissements d'enseignement et de recherche français ou étrangers, des laboratoires publics ou privés. 


\title{
ELECTROMAGNETIC FLUCTUATIONS IN THE VICINITY OF THE MAGNETOPAUSE
}

\author{
L. Rezeau, S. Perraut, and A. Roux
}

Centre de Recherches en Physique de l'Environnement, CNET 92131 Issy-les-Moulineaux, France

\begin{abstract}
A detailed analysis of electromagnetic fluctuations recorded during several magnetopause and boundary layer crossings by the ESA GEOS-2 geostationary spacecraft is presented. A high level of electromagnetic $\left(\delta \mathrm{E} / \delta \mathrm{B} \sim \mathrm{V}_{\mathrm{A}}\right)$ fluctuations in the ULF $(0-10 \mathrm{~Hz})$ range is observed for each such crossing. The obtained frequency spectra are shown to fit with a power law $\mathrm{B}^{2} \sim \mathrm{f}^{-\alpha}$. The distribution of these $\alpha$ indexes is found to be narrow (standard deviation $\leqslant 0.25$ for $0.5 \leqslant f \leqslant 11 \mathrm{~Hz}$ ), with a peak value $\alpha \sim 2.5$ which does not depend on the direction with respect to the magnetic field. Data suggest that the magnetopause boundary layer is the source of these intense fluctuations.
\end{abstract}

\section{Introduction}

It has been shown that a boundary layer of dense plasma exists all over the magnetopause on the earthward side (Eastman and Hones, 1979; Paschmann et al, 1979). Since this layer is populated by magnetosheath particles, together with a typical magnetospheric plasma, and since it always exists, there must be some ways by which the magnetosheath particles can penetrate through the magnetopause, whatever the solar wind conditions. Tsurutani and Thorne (1982) have investigated the cross-field diffusion of magnetosheath ions as a possible way to explain the existence of the magnetopause boundary layer. While concluding that diffusion by resonant fluctuations is a likely cause, they were not able to identify the relevant turbulence. Gendrin (1983) challenged their conclusion that electrostatic waves should be operative and showed that the magnetic fluctuations reported by Perraut et al (1979) have a sufficient level for insuring the required rate of cross-field diffusion. We further investigate this process by using electric field and magnetic field data collected on GEOS 2, during several magnetopause crossings. This allows to characterize the statistical properties of the spectra of observed magnetic fluctuations. Indeed, in view of the large level of the fluctuations and of their random nature, it seems convenient to use methods similar to those developed for studying fluid turbulence and to compare to power laws. The well known $\mathrm{k}^{-5 / 3}$ law for hydrodynamic turbulence has been established by Kolmogorov (1941); later Kraichnan (1965) showed that a $k^{-3 / 2}$ law holds in the case of incompressible magnetohydrodynamics. These spectral laws are based upon dimensional analysis for which the assumptions of isotropy and homogeneity are mandatory. It is interesting to compare these laws with experimental data (Montgomery, 1985). We have made a systematic study of ULF fluctuations during several single or multiple magnetopause crossings which occurred on August 28 and September 29,

Copyright 1986 by the American Geophysical Union.

Paper number 6L6289.

0094-8276/86/006L-6289\$03.00
1978. We first compute the integrated power of the fluctuations from 0.5 to $10 \mathrm{~Hz}$, and then fit power spectra with $f^{-\alpha}$ spectral laws.

\section{GEOS Observations}

The three-component search coil magnetometer onboard the GEOS 2 geostationary satellite covers a frequency range up to $11.5 \mathrm{~Hz}$, in wave form. The $\mathrm{BZ}$ component is aligned with the spin axis and hence does not suffer from any effect induced by the spacecraft rotation. Rejection of spurious signals at the spin frequency on orthogonal components is achieved onboard by a filter working at the spin frequency $(T \sim 6$ s). Data processing is described by Kodera et al. (1977) and Robert et al (1979). The GEOS 2 magnetic antennas are rather sensitive in a broad frequency range, namely $10^{-2}$ $\mathrm{nT} . \mathrm{Hz}-\mathrm{T} / 2$ at $0.5 \mathrm{~Hz}$ and $10^{-3} \mathrm{nT} . \mathrm{Hz}^{-1 / 2}$ at $3 \mathrm{~Hz}$.

This wave experiment in the Ultra Low Frequency range has been continuously operating. During periods of large magnetic activity, GEOS 2 has experienced multiple magnetopause crossings in the morning and dayside sectors. Very strong fluctuations covering the whole frequency range have been detected at the magnetopause crossings and in their vicinity (Perraut et al, 1979). The present study has been carried out from data of August 28, 1978 (from 500 to 1000 UT) and September 29, 1978 (from 600 to 1100 UT). During these days, the magnetopause and its boundary layer were crossed 19 times.

\section{Integrated Power of the Fluctuations}

Figure 1 shows a one-hour period selected among the various magnetopause crossings. The integrated power of the magnetic components is plotted with a time resolution of $22 \mathrm{~s}$ and between 0.5 and $10 \mathrm{~Hz}$ (the lower limit is about three times the spin frequency to avoid any remaining spurious effect due to the spacecraft rotation) and it is shown together with the VDH components of the DC magnetic field. The magnetopause is determined from the $\mathrm{BH}$ turnover and the boundary layer from the spacecraft potential (Knott et al, 1983). It is clear that the integrated magnetic power is the most intense at the magnetopause crossings (up to $100 \mathrm{nT}^{2}$ ), when the $\mathrm{DC}$ magnetic field decreases down to $60 \mathrm{nT}$, which corresponds to

$\delta \mathrm{B} / \mathrm{B} \sim 15 \%$. In the boundary layer, the power is similar to that observed in the magnetopause (1-100 $\left.\mathrm{nT}^{2}\right)$. In the magnetosheath, the level is lower $\left(1-10-\mathrm{nT}^{2}\right)$ and still much lower in the magnetosphere $\left(10^{-2}-10^{-1} \mathrm{nT}^{2}\right)$. The observed large decrease in the level of the fluctuations as the spacecraft moves away from the magnetopause and its boundary layer suggests the existence of a source in the magnetopause and boundary layer. This cannot, however, be directly tested since we have no way of measuring the distance between the spacecraft and the magnetopause. 
GEOS- 2 AUG. 28,1978 05.00UT
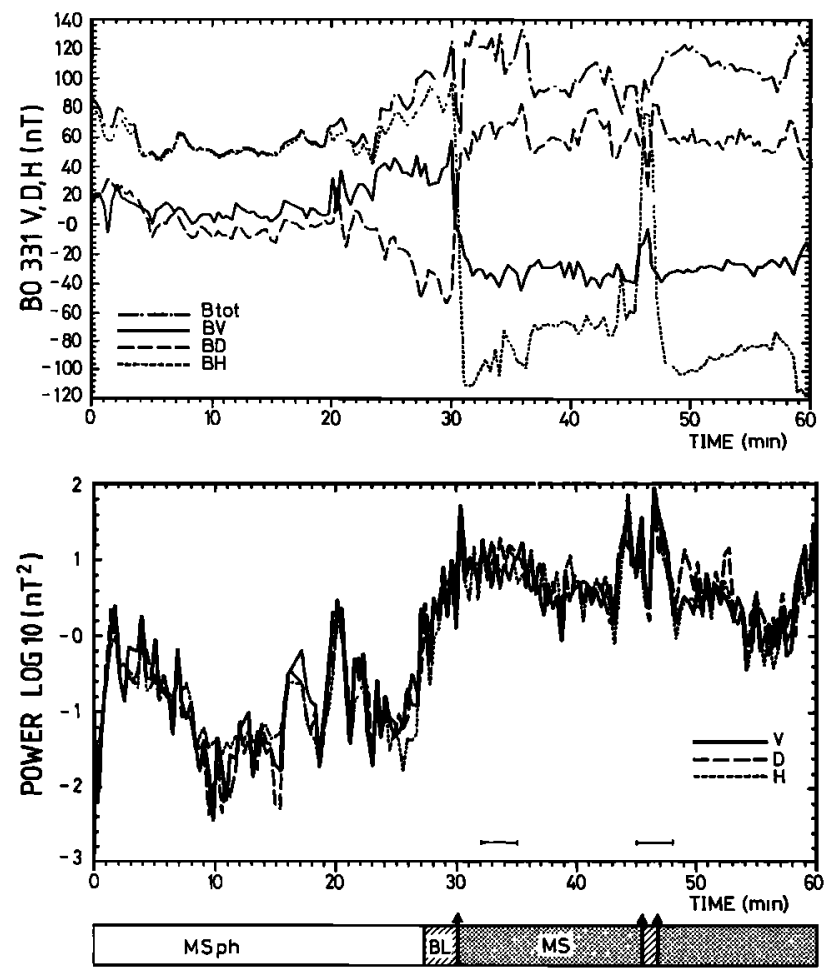

Fig. 1. DC magnetic field variations (upper panel) and power of the magnetic signal integrated over $22 \mathrm{~s}$ between 0.5 and $10 \mathrm{~Hz}$ (lower panel) on August 28,1978 , in the VDH coordinate system. Location of GEOS 2 in the magnetosphere (MSph), the magnetosheath (MS) and in the boundary layer (BL) are indicated at the bottom. Magnetopause crossings (arrows) are determined from reversal in $\mathrm{BH}$. Also represented are the time intervals (horizontal bars in the lower panel), over which a Fourier analysis of fluctuations has been performed (see Fig. 3 and 4).

\section{Spectra of Magnetic Components}

In Figure 2, the wave form of electric and magnetic field fluctuations are presented around 0544:20. The typical ratio $\delta \mathrm{E} / \delta \mathrm{B}$ is of the order of $V_{A}$, the Alfven velocity. They are very intense and Show a very erratic behaviour; both features are not consistent with what is expected for a wave. Thus it seems more appropriate to plot the spectra of magnetic components. It is difficult to proceed in the same way for the electric component indeed, because a failure of a solar array has led to truncate the electric field data, as indicated in Figure 2 by horizontal segments. Magnetic spectra are computed over $22 \mathrm{~s}$, and then 9 successive spectra are averaged $(3 \mathrm{~min}$ data). The spectra displayed in Figure 3 show a rather smooth decrease of the averaged power spectral density versus frequency, consistent with what is expected for a turbulent spectrum. This suggests to fit these spectra with power laws; for this purpose, we have used a least squares method. For each spectrum, we have determined the index $\alpha$ and the power $\rho$ at $1 \mathrm{~Hz}$, so that $\mathrm{P}=\rho_{\mathrm{f}} \mathrm{f}^{-\alpha}$ $P$ being the power spectral density. The parameter $\Delta=\frac{1}{N} \sum_{i=1}^{N}\left|\log \mathrm{P}_{\mathrm{i}}-\log \rho_{\mathrm{o}}+\alpha \log \mathrm{f}_{\mathrm{i}}\right|$ indicates the scattering of the spectrum around the fitting curve
(Figure 4). The error on $\alpha$ is deduced from $\Delta$ by $\delta \alpha=\Delta /\left(\log f_{\max }-\log f_{\min }\right)$. This has been done in two frequency ranges: from $0.5 \mathrm{~Hz}$ to $3 \mathrm{~Hz}$ and from $0.5 \mathrm{~Hz}$ to $11 \mathrm{~Hz}$. We have studied 29 spectra on August 28, 1978, and 15 on September 29, 1978, in two coordinate systems. In the first case, $B Z$ is aligned with the DC magnetic field, BL and BR are the left and right-hand components projected in the plane perpendicular to it; the other coordinate system is the standard VDH coordinate system. The reason for displaying the data in various coordinate systems was to find whether there is any preferred direction for the magnetic field fluctuations. Two spectra are plotted in Figure 3, the time intervals when they were taken are indicated in Figure 1. During the first one, GEOS 2 was in the magnetosheath, while during the second one, it crossed the magnetopause twice.

As can be seen for instance in Figure 3, the power law found from the least squares method happens to depend upon the region where the spectra were made, then we have split them into three classes, namely (i) magnetopause or boundary layer (19 cases), (ii) magnetosheath (14 cases), and (iii) magnetosphere (11 cases). The level of turbulence is so high during the boundary crossings that it is dominant even if they last less than the three minutes sampled. In each region, we studied the statistical distribution of the indexes $\alpha$.The results are given in Table 1: for each region, frequency range, and component, we calculated the average value of the index $\langle\alpha\rangle$, the average error $\langle\delta \alpha\rangle$ and the standard deviation $\sigma$ of the distribution of the indexes $\alpha$.

These results allow to draw some interesting conclusions: - first, it is clear that the fit with a power law works pretty well, especially in the case of the magnetopause and boundary layer; indeed, both the average error and the standard deviation are small. In order to assess the validity of the determination of the index $\alpha$, it is interesting to compare the parameters $\langle\delta \alpha\rangle$ and $\sigma$. In the magnetopause and boundary layer, $\sigma$ is close to $\delta \alpha$, which means that the fit with a single

GEOS-2 AUG. 28, $1978 \quad S-300$
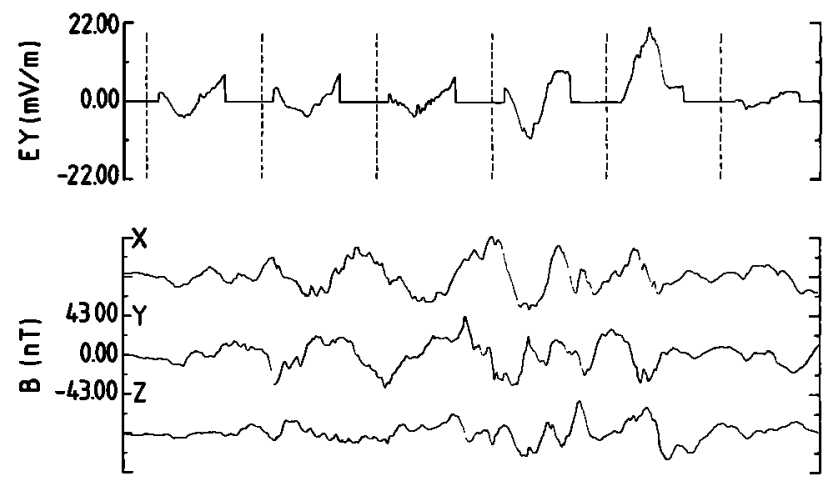

\section{$5: 44.10-5: 4420-54430$}

Fig. 2. Electric (upper curve) and magnetic field waveforms in a frame rotating with the satellite, $Z$ being aligned with the spin axis. The electric antenna is directed towards the Sun at the times corresponding to the vertical dotted lines; data are truncated because of a failure of a solar array. 
GEOS-2 AUG.28,1978 S-300
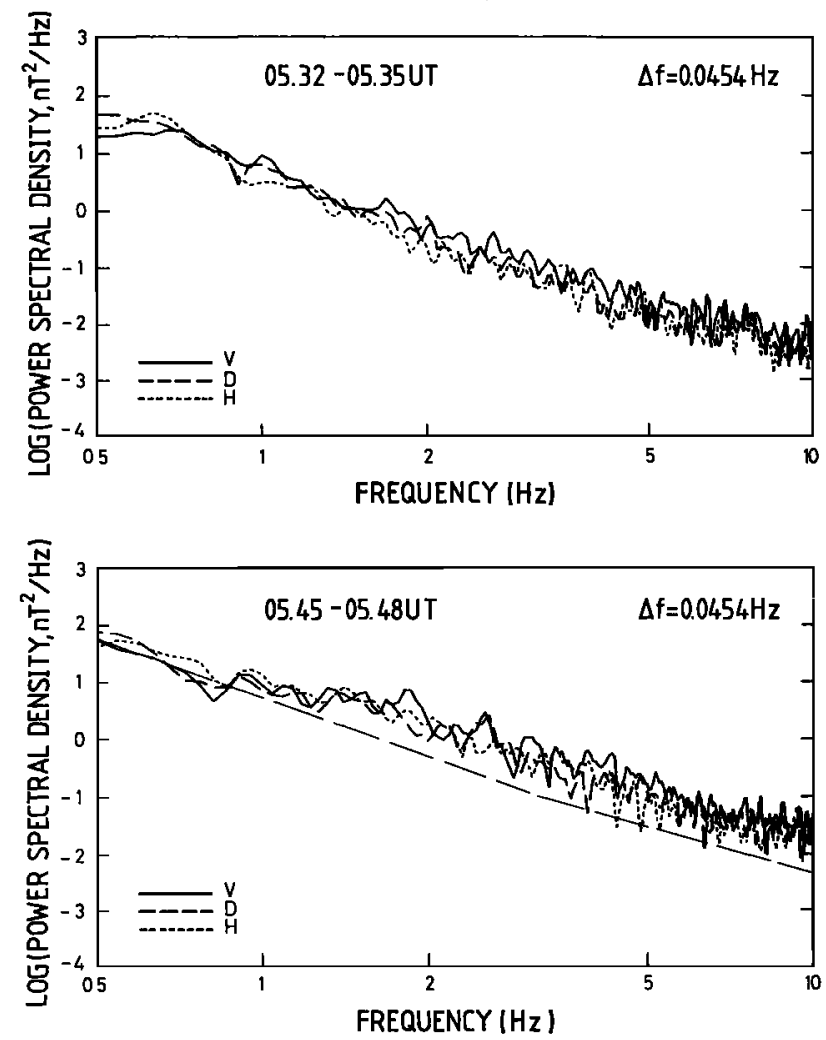

Fig. 3. Spectra of the magnetic fluctuations in the magnetosheath (upper panel), and in magnetopause and boundary layer crossings (lower panel). The dot dashed line shows the average of the upper spectra for comparing the slope of these spectra.

value of $\alpha$ is appropriate within the accuracy of the determination of $\alpha$. In the magnetosheath and magnetosphere, the standard deviation largely exceeds the average error, thus implying that fitting with a power law with a single index is not possible.

- second, for the three components corresponding to the same frequency range and region, the intervals $\langle\alpha\rangle \pm\langle\delta \alpha\rangle$ overlap, thus the spectral index

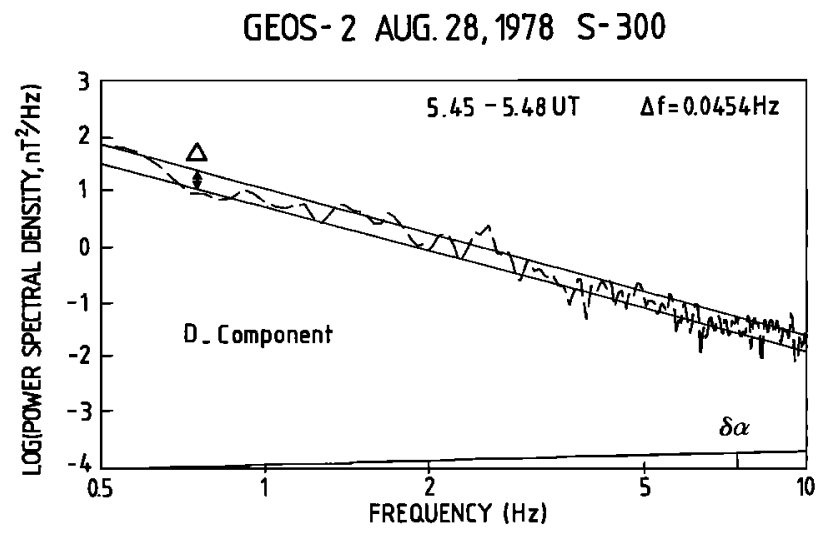

Fig. 4. Fit of the D-component spectrum with a P $=\rho_{\mathrm{f}^{-\alpha}}$ law. $\alpha=2.71, \rho_{0}=9.1 \mathrm{nT}^{2} \mathrm{~Hz}^{-1}$, $\Delta=0.16$. The two curves correspond to the extremum law. The error on the index, $\delta \alpha$ is visualized at the bottom of the figure. $\alpha$ is isotropic. It must be noticed, however, that the coefficient $\rho_{\circ}$ is not always isotropic: in the magnetosphere and ${ }^{\circ}$ deep magnetosheath, the power is often lower on the $Z$ component. Besides, there is no significant difference between the two coordinate systems, which confirms that fluctuations are isotropic.

-third, in the boundary regions, there is an overlapping of the intervals $\langle\alpha\rangle \pm\langle\delta \alpha\rangle$ in the two frequency ranges $(0.5-3 \mathrm{~Hz}$ and $0.5-11 \mathrm{~Hz})$, which strengthens our conclusion that in these regions, the spectra can be fitted with an $f^{-\alpha}$ power law.

In the magnetosphere, the standard deviation of the results is much larger, thus no $\mathrm{f}^{-\alpha}$ spectral law seems to apply. This is not much surprising, since the level of the fluctuations is very low. In the magnetosheath, it is more difficult to conclude, because in both frequency ranges there is a quite good agreement with a $\mathrm{f}^{\alpha}$ law, yet index $\alpha$ changes with the frequency range: in the low frequency part, the decrease is much faster $\left(f^{-3.6}\right)$ than in the high one $\left(f^{-3}\right)$. How can we explain the different behaviour of the various parts of the magnetosheath spectra? As mentioned above, the fluctuation levels in the magnetosheath are lower, thus suggesting that the magnetopause and boundary layer are the source of the fluctuations observed in the magnetosheath. Furthermore, this can explain the different frequency dependences in the two media; indeed the propagation of the fluctuations from their source being frequency-dependent, one expects the lower frequencies (larger wave lengths) to propagate further out than the higher frequencies (lower wave lengths ). This frequency-dependent attenuation can explain first the larger index obtained in the magnetosheath, and second, the fact that this index decreases from low frequencies to high ones. Thus these frequency-dependent laws are consistent with the assumption that the magnetopause is the source of the fluctuations.

Table 1. Computed values of $:<\alpha>$ average value of indexes $\alpha,\langle\delta \alpha\rangle$ average error on $\alpha, \sigma$ standard deviation of the distribution of $\alpha$. 0.5 to $3 \mathrm{~Hz} 0.5$ to $11 \mathrm{~Hz}$

\begin{tabular}{lcccccc} 
& $\langle\alpha\rangle$ & $\langle\delta \alpha\rangle$ & $\sigma$ & $\langle\alpha\rangle$ & $\langle\delta \alpha\rangle$ & $\sigma$ \\
\hline BL & 2.52 & 0.23 & 0.26 & 2.59 & 0.14 & 0.22 \\
BR & 2.45 & 0.21 & 0.31 & 2.63 & 0.13 & 0.19 \\
BZ & 2.44 & 0.23 & 0.41 & 2.48 & 0.14 & 0.20 \\
V & 2.28 & 0.23 & 0.37 & 2.49 & 0.14 & 0.22 \\
D & 2.57 & 0.21 & 0.40 & 2.64 & 0.13 & 0.13 \\
H & 2.49 & 0.22 & 0.36 & 2.55 & 0.15 & 0.24 \\
& & \multicolumn{7}{c}{ MAGNETOSHEATH } & & \\
BL & 3.76 & 0.18 & 0.57 & 3.02 & 0.13 & 0.34 \\
BR & 3.45 & 0.17 & 0.37 & 3.02 & 0.13 & 0.38 \\
BZ & 3.43 & 0.18 & 0.50 & 2.88 & 0.14 & 0.32 \\
V & 3.45 & 0.18 & 0.45 & 2.92 & 0.13 & 0.32 \\
D & 3.73 & 0.19 & 0.52 & 3.07 & 0.15 & 0.38 \\
H & 3.61 & 0.17 & 0.43 & 3.00 & 0.13 & 0.36 \\
& \multicolumn{7}{c}{ MAGNETOSPHERE } & & \\
BL & 3.54 & 0.25 & 0.96 & 2.91 & 0.21 & 0.54 \\
BR & 3.32 & 0.23 & 0.97 & 2.87 & 0.19 & 0.54 \\
BZ & 3.26 & 0.25 & 0.64 & 2.59 & 0.19 & 0.66 \\
V & 3.64 & 0.26 & 0.83 & 2.89 & 0.19 & 0.48 \\
D & 3.32 & 0.25 & 1.13 & 2.81 & 0.20 & 0.54 \\
H & 3.43 & 0.25 & 0.70 & 2.60 & 0.19 & 0.70 \\
\hline
\end{tabular}




\section{Summary and Discussion}

During two days where the solar wind ram pressure was so large that GEOS-2 happened to cross the magnetopause several times, a detailed study of ULF fluctuations has been carried out. It has been shown that (i) the power spectral density in the magnetopause boundary layer can be fitted by a power law $\mathrm{f}^{-\alpha}$ with a single value of $\alpha \simeq 2.5$ for all crossings or all components, (ii) by applying the same procedure to magnetosheath spectra, we found a different index $\alpha$, but both the standard deviation and the difference between the values of $\alpha$ obtained for each component exceed the uncertainties. Furthermore, $\alpha$ depends upon the frequency range considered $(\alpha \simeq 3.5$ for $0.5 \leq \mathrm{f} \leq 3 \mathrm{~Hz}$ and $\alpha \simeq 3$ for $0.5 \leq \mathrm{f} \leq 11 \mathrm{~Hz}$, respectively). In the magnetosphere itself, the power spectral density is by 2 or 4 orders of magnitude weaker; furthermore, a fit with a $f^{-\alpha}$ law is irrelevant. Both the much higher level of fluctuations observed in the magnetopause boundary layer and the change in the spectral laws from magnetopause to magnetosheath are consistent with a source in the magnetopause and its boundary layer. Definitive proof of this, however, cannot be established from single spacecraft measurements.

Since the fluctuations observed in the magnetopause boundary layer are very intense (up to $\delta \mathrm{B} / \mathrm{B} \sim 15 \%)$, random, and can be fitted with a single power law, it is tempting to interpret them as a turbulent spectrum. The obtained index $\alpha=2.5$ is quite different from $\alpha=5 / 3$ and $\alpha=$ $3 / 2$ found by Kolmogorov (1941) and by Kraichnan (1965), respectively. It should, however, be stressed that neither of these theoretical results apply to the strongly magnetized plasma considered here. Furthermore, the above authors have obtained, by dimension analysis, a power law in $k$ space which does not necessarily imply a power law with the same index in frequency space. This problem has been solved by Matthaeus and Goldstein (1982), who took advantage of the super-Alfvenic speed of the solar wind flow and assumed isotropy for converting the frequency spectra observed onboard a single spacecraft into a spectrum in $k$ space. We are not a priori in a position to do so.

Given the high level of magnetic fluctuations in the magnetopause and its boundary layer, it is worth investigating their potential role in the spatial diffusion of the plasma through this boundary. From the expressions given by Tsurutani and Thorne (1982) by using a sequence of GEOS data, Gendrin (1983) concluded that the rate of diffusion he obtained (D $\sim 400 \mathrm{~km}^{2} . \mathrm{sec}^{-1}$ ) is sufficient for explaining the existence of a boundary layer adjacent to the magnetopause. While our study is in general agreement with this conclusion, we feel it necessary to take into account the dependence $\left(\mathrm{f}^{-\alpha}\right)$ of the observed spectra in the evaluation of the diffusion rate; this will be done elsewhere.
Acknowledgements. We are pleased to acknowledge A. Pedersen (Space Science Department, ESTEC, The Netherlands), who kindly provided us with electric field data.

\section{References}

Eastman, T.E., and E.W. Hones, Characteristics of the low latitude boundary layer and magnetopause layer at high time resolution, J. Geophys. Res., 84, 2019-2028, 1979.

Gendrin, R., Magnetic turbulence and diffusion processes in the magnetopause boundary layer, Geophys. Res. Lett., 10, 769-771, 1983.

Knott, K., P. Décréau, A. Korth, A. Pedersen, and G.L. Wrenn, Observations of the GEOS equilibrium potential and its relation to the ambient energy distribution, ESA/SP-198, 19 - 24, 1983.

Kodera, K., R. Gendrin, and C. de Villedary, Complex representation of a polarized signal and its application to the analysis of ULF waves, $\mathrm{J}$. Geophys. Res., 82, 1245-1255, 1977.

Kolmogoroff, A.N., The local structure of turbulence in incompressible viscous fluid for very large Reynolds numbers, C.R. Acad. Sci. URSS, $30,301,1941$.

Kraichnan, R.H., Initial-range spectrum of hydromagnetic turbulence, Phys. Fluids., \& 1385, 1965.

Matthaeus, W.H., and M.L. Goldstein, Measurements of the rugged invariants of magnetohydrodynamic turbulence in the solar wind, J. Geophys. Res., $87,6011,1982$.

Montgomery, D., Characteristic phenomena of magnetohydrodynamic turbulence, in Future Missions in Solar, Heliospheric and Space Plasma Physics, ESA/SP-235, pp. 175-179, 1985.

Paschmann, G., N. Sckopke, G. Haerendel, J. Papamastorakis, S.J. Bame, J.R. Asbridge, J.T. Gosling, E.W. Hones, Jr., and E.R. Tech, ISEE plasma observations near the subsolar magnetopause, Space Sci. Rev., 22, 717-737, 1979.

Perraut, S., R. Gendrin, P. Robert, and A. Roux, Magnetic pulsations observed onboard GEOS-2 in the ULF range during multiple magnetopause crossings, ESA/SP-148, $113-122,1979$.

Robert, P., K. Kodera, S. Perraut, R. Gendrin, and C. de Villedary, Amplitude et polarisation des ondes UBF détectées à bord du satellite GEOS-1. Méthodes d'analyse, problèmes rencontrés et solutions pratiques, Ann. Telecom., 34, 179-1 86, 1979.

Tsurutani, B.T., and R.M. Thorne, Diffusion processes in the magnetopause boundary layer, Geophys. Res. Lett., 9, 1247-1250, 1982.

L. Rezeau, S. Perraut and A. Roux, CNET/CRPE, 92131 Issy-les-Moulineaux, France.

(Received July 17, 1986; revised August 28, 1986; accepted September $\overline{25}, 1986$. 